\title{
Article
}

\section{Training Preferences of School Food Service Staff Vary by Role in the School Nutrition Program}

\author{
Leia Flure ${ }^{1, *}$, Melissa Pflugh Prescott ${ }^{2}$, Whitney Ajie ${ }^{3}$, Trinity Allison ${ }^{1}$ and Jennifer McCaffrey ${ }^{1}$ \\ 1 Office of Extension and Outreach, University of Illinois at Urbana-Champaign, Urbana, IL 61801, USA; \\ trinitya@illinois.edu (T.A.); jmccaffr@illinois.edu (J.M.) \\ 2 Department of Food Science and Human Nutrition, University of Illinois at Urbana-Champaign, Urbana, \\ IL 61801, USA; mpp22@illinois.edu \\ 3 Washington State Department of Health, Tumwater, WA 98501, USA; whitney.ajie@doh.wa.gov \\ * Correspondence: lweston2@illinois.edu
}

check for updates

Citation: Flure, L.; Pflugh Prescott, M.; Ajie, W.; Allison, T.; McCaffrey, J. Training Preferences of School Food Service Staff Vary by Role in the School Nutrition Program. Int. J. Environ. Res. Public Health 2021, 18, 50.

https://dx.doi.org/10.3390/ ijerph18010050

Received: 31 October 2020

Accepted: 21 December 2020

Published: 23 December 2020

Publisher's Note: MDPI stays neutral with regard to jurisdictional claims in published maps and institutional affiliations.

Copyright: () 2020 by the authors. Licensee MDPI, Basel, Switzerland. This article is an open access article distributed under the terms and conditions of the Creative Commons Attribution (CC BY) license (https: / / creativecommons.org/ licenses/by/4.0/).

\begin{abstract}
Professional development has been identified as a critical component for school nutrition professionals (SNPs) to successfully implement school meal standards in the United States. However, training needs may vary based on different factors. This study examined (1) the topics of highest priority for SNPs; (2) preferred learning methods; (3) where and when trainings should be conducted; and (4) whether responses differ according to important factors including position type, school locale (urban vs. rural), or job experience. Participants completed surveys that included questions on demographics and preferences for learning methods and training topics $(n=492)$. Descriptive statistics characterized survey responses. Chi square tests assessed differences in learning method and training topic preferences by participant role, locale, and job experience; Cramer's V assessed the strength of association for each chi square result. Qualitative responses to open-ended questions were analyzed using an inductive thematic analysis method. Nearly all training topic preferences were significantly different $(p<0.001$ using Bonferroni method) when stratified by role. Significant differences were also observed for school locale and years of experience, but to a lesser degree. There was less variation in learning method preferences across staff role. Qualitative results $(n=93)$ identified three key themes related to training needs: role-specific trainings, innovative learning methods, and geographic access. The combination of quantitative and qualitative analysis indicate that professional development for SNPs should mostly be conducted in-person, be easily accessible, and include hands-on activities. Further, training should be tailored by job role and address situational barriers unique to the geographic area.
\end{abstract}

Keywords: National School Lunch Program; nutrition; school feeding programs; school nutrition success

\section{Introduction}

In 2010, the United States federal government updated standards for foods served in child nutrition programs through the Healthy, Hunger Free Kids Act [1]. As a result of these changes, school nutrition professionals (SNPs) have faced significant challenges. Past surveys have indicated that a large proportion of SNPs have difficulty understanding the guidelines, which included increased portion sizes for fruits and vegetables and nutrient limits, among other changes. SNPs have also struggled with barriers to implementation, such as workforce retention and lack of equipment for scratch cooking [2]. Other concerns have included menu planning with tightened sodium limits [3-5], poor student acceptance of whole grain foods and vegetables [6-10], and lack of culinary skills for serving palatable foods that meet nutrition guidelines [3]. Correspondingly, multiple surveys have identified professional development as a key need for SNPs to successfully implement federal rules for school meals [2-4].

To strengthen the administration of school nutrition programs, the United States Department of Agriculture (USDA) defined minimum hiring criteria for school nutrition 
directors and established continuing education requirements for SNPs, known as the USDA Professional Standards [11]. In effect since 2015, the USDA Professional Standards outline annual training needs in hours, with requirements varying according to a person's role in the school nutrition program. For example, school nutrition directors must complete at least $12 \mathrm{~h}$ of annual continuing education while part time staff working less than $20 \mathrm{~h}$ per week require four hours annually. Trainings must be aligned with specific learning topics and objectives delineated by the USDA and importantly, should be relevant to the employee's job duties. A cashier would be expected to attend trainings on topics like customer service and identifying reimbursable meals, but not topics more suited for a cook, such as knife skills or standardized recipes.

Several studies shed light on SNPs' training needs based on knowledge, skills, and attitudes for predetermined topics; these include food allergies and other special dietary needs [12-16], whole grains [6,7,17], school food environment [18], farm to school [19], food hygiene and safety practices [20], and scratch cooking [21]. The few studies asking SNPs specifically about their training needs have indicated a desire for assistance with menu planning, with attention to meeting new requirements for nutrition limits and portion sizes [22,23]. Within the studies reviewed, food service directors, managers, and upper level administrators were the primary respondents $[19,22]$; they were either specifically targeted or there were few responses from food service workers such as cooks, servers, and cashiers. In the latter case, supervisors reported perceived training needs for their staff [22].

Overall, the limited research regarding food service workers' topic preferences suggests they desire training in line with their job duties (e.g., cooking skills, food safety, meal pattern requirements) [23]. However, there are other factors to take into account when planning training topics for SNPs. Geographic area should be considered as it can affect resource availability [24,25]. In rural areas, for example, access to certain products is often limited and food costs may be higher as they order in smaller volumes $[5,17,25-27]$. Thus, a focus on procurement strategies could be helpful to address these barriers.

Learning methods, too, should be considered based on these factors. Face-to-face team building and skills workshops may be particularly helpful for frontline staff-especially those who work part-time and may be less experienced-to build confidence, enhance motivation, and increase the likelihood of putting knowledge and skills into practice [21,28,29]. Other learning methods such as online courses may be preferable for some learners, but to our knowledge, there is no research investigating this question specific to SNPs. Online training may also not work well for SNPs in rural areas where internet infrastructure issues cause poor connectivity [5,17,24-27].

Regardless of factors like position or location, attendance barriers such as timing and limited funds for travel and additional pay are perennial concerns $[13,18,29]$. Further, the lack of time for training may be worsened by labor shortages and the burdens of meal planning, preparation, and documentation [2]. It is imperative to identify the most opportune logistics to maximize attendance while minimizing strain on school nutrition programs.

Increasing participation in professional development tailored according to important factors may improve SNPs' ability to provide school meals that meet federal standards and positively impact student nutrition. The aims of this study were to explore (1) what topics are of highest priority for school nutrition professionals; (2) what learning methods are most preferred; (3) where and when trainings should be conducted; and (4) whether responses differ according to position type, school locale, or job experience. These results will help inform planning and development of current and future training programs for SNPs.

\section{Materials and Methods}

In 2015, the Illinois State Board of Education (ISBE) contracted with University of Illinois Extension and Outreach (Extension; part of the national cooperative extension service) to supplement their support of school nutrition programs. This partnership, 
named the ABCs of School Nutrition program, built infrastructure to provide ongoing statewide training opportunities. As part of the formative evaluation, Extension and ISBE conducted a survey to gather information on the training and learning preferences of Illinois school staff with child nutrition related job duties. At a later date, details about the survey and data collection were submitted to the University of Illinois Office for the Protection of Research Subjects. The study was granted a Not Human Subjects Research Determination, allowing the research team to analyze data and disseminate results without additional Institutional Review Board approval.

\subsection{Survey Design}

The survey was drafted by the research team with expert review from the Illinois School Nutrition Association and ISBE's nutrition and wellness staff. Questions included items on demographics, learning method preferences, and training preferences (topics, timing, length).

\subsubsection{Demographics}

Participants were asked to select from the following role types: Food service director (FSD); food service managerial (FSM-head cook, kitchen manager, etc.); all other food service staff [food service worker (FSW); server, cashier, cook, etc.]; school administration (superintendent, principal, business manager); other school staff (nurse, teacher, secretary, etc.).

Participants were given the option to disclose which school district(s) they work with. If they chose to do so, the research team matched the district name with ISBE data for number of students, number of schools, and free- and reduced-price lunch eligibility rates. Participants who did not wish to disclose their district name were presented with questions that asked them to estimate this information. For example, they could select $40 \%$ or lower, $41-59 \%, 60 \%$ or higher, or "I'm not sure" regarding the percentage of students eligible for free- and reduced-price lunch.

Each respondent that identified their school district was categorized as working in a rural or urban/suburban location based on publicly available information from the National Center for Educational Statistics website [30]. School districts with locale codes 11-13 and 21-23 in the database were categorized as urban/suburban while those with codes $31-33$ and $41-43$ were categorized as rural.

\subsubsection{Learning Method Preferences}

Participants were asked about their preferences for different learning methods and activities, such as working in groups versus with a partner versus alone; role play or demonstrations, problem solving, or teaching others the information; online vs. face to face; etc. These items were drafted based on existing best practice information for adult learning [31] as well as feedback from in-person ABCs of School Nutrition training evaluations, and feedback from Extension educators who facilitated trainings. Items followed a Likert scale where 1 = dislike a great deal, 2 = dislike somewhat, $3=$ neither like nor dislike, $4=$ like somewhat, and $5=$ like a great deal.

\subsubsection{Training Timing and Length}

Regarding timing, respondents were asked to select their preferred training times by the month, day of the week, and time of day [early morning (7-10 am); later morning (10-12 pm); early afternoon (12-2 pm); later afternoon (2-4 pm)]. They were able to choose multiple preferred options for each of these. Participants were also asked about preference for length of a single training (less than one hour; $1-2 \mathrm{~h} ; 2-4 \mathrm{~h} ; 4 \mathrm{~h}$ or more) and could select multiple options. 


\subsubsection{Training Topics}

Training topic questions were divided into four sections to align with the USDA Professional Standards: Nutrition, Operations, Administration, and Communications \& Marketing [32]. Participants rated how likely they would be to attend training on various topics in each category (very unlikely; somewhat unlikely; neither likely or unlikely; somewhat likely; very likely). Examples included the following: Portion sizes, reimbursable meal requirements (Nutrition); production records, procurement strategies (Operations); financial management, assessing and planning for equipment needs (Administration); food merchandising techniques, using social media to promote child nutrition programs (Communications \& Marketing).

\subsubsection{Incentives}

At the end of the survey, respondents were given the opportunity to be entered into a drawing to win one of three school nutrition banners valued at up to $\$ 200$.

\subsection{Participant Recruitment and Survey Distribution}

\subsubsection{Recruitment}

The research team did not aim for a specific number of participants, but promoted the survey to the audience of focus with the goal of collecting as many survey responses as possible within a predetermined time period of approximately 3 months (June, July, and August).

\subsubsection{Online Survey}

An online version of the survey was built through the Qualtrics web platform (Qualtrics, Provo, UT). The survey link was distributed through multiple channels, including ISBE's monthly "The Outlook" e-newsletter (sent to approximately 1200 school nutrition program sponsors and 600 others who opt in) and the ABCs of School Nutrition monthly "On Your Tray" e-newsletter, which was sent to about 250 SNPs, organizational partners, and other subscribers. The link was also shared throughout the summer of 2018 on the ABCs of School Nutrition Facebook page. Printed cards with the survey link were given out to SNPs attending the Illinois School Nutrition Association and ISBE conferences in June and August 2018.

\subsubsection{Paper Survey}

SNPs who attended professional development events led by Extension educators were given the opportunity to fill out paper versions of the survey on site. Data from the paper surveys were entered into Qualtrics manually and denoted as such to distinguish them from data obtained through the online version.

\subsection{Inclusion/Exclusion Criteria}

The primary target audience for the survey was school staff with job responsibilities related to child nutrition programs. The first question of the survey was "In your current role, do you have any duties or responsibilities related to child nutrition programs at your school or district?" Those who answered "no" were exited from the online version of the survey. Data were not included from any school employees without related job duties who might have completed the paper survey despite instructions to the contrary. Although the survey was intended for school staff within Illinois, it was not a requirement for participation.

\subsection{Statistical Analyses}

Data were analyzed with Stata (version 15.1, 2017, https:/ /www.stata.com/). Descriptive statistics were used to characterize survey responses. Chi square tests were used to assess differences in learning method and training topic preferences by participant role, locale (urban vs rural), and job experience. Cramer's V was used to assess the strength of 
association for each chi square result, with $<0.20$ as weak, $0.20 \leq X<0.30$ as moderate, and $\geq 0.30$ as strong [33]. For each chi square analysis, the 5-point Likert scale was collapsed into three categories. The collapsed categories were (1) dislike a great deal or dislike somewhat, (2) neither like/dislike, and (3) like somewhat or like a great deal for learning method preferences, and they were (1) very unlikely or somewhat unlikely, (2) neither likely nor unlikely, and (3) somewhat likely or very likely for training topic preferences. Due to the large number of statistical tests used, the Bonferroni method was used to reduce the likelihood of type 1 error and $\alpha<0.05 / 50=0.001$ was used to infer significance.

\subsection{Qualitative Analyses}

Participant responses to the open-ended question, "In your own words, are there other important aspects of your training needs that you want to share?" were categorized by participant role and analyzed by a single researcher using an inductive thematic analysis method [34-36]. Themes were only included if they occurred across at least 4 of the 5 participant role categories and the number of responses for the theme constituted at least $10 \%$ of the total qualitative sample. Some participants provided responses that encompassed more than one theme.

\section{Results}

A total of 492 people participated in the survey. There was likely overlap among recruitment outlets where prospective participants would have viewed recruitment messages; therefore, a response rate was not calculated due to the possibility of duplication errors.

Some questions were not applicable to all survey participants and some participants chose not to answer some questions. Subsequently, the sample size for the training topic and learning method preferences ranged from 453 to 461 , and the range for school demographic questions was 115 to 446 . As shown in Table 1, FSDs $(32.7 \%, n=161)$ were the most common staff to participate in the survey, and almost $90 \%$ of participants completed the survey online $(n=446)$. The majority of SNPs completing the survey worked in urban areas $(63.6 \%, n=232)$ and had 501-2499 students in their district $(50.4 \%, n=58)$. Survey submission method (online vs. paper) significantly varied by staff role $\left(\chi^{2}(3)=181.9\right.$, $p<0.0001$, Cramer's V $=0.61$ ). FSWs comprised $53 \%$ of the paper survey submissions.

Table 1. Characteristics of the Training \& Learning Preferences Survey for Illinois Child Nutrition Program Survey participants.

\begin{tabular}{lc}
\hline \multicolumn{1}{c}{ Characteristics } & Frequency (\%) \\
\hline Role in child nutrition: $(n=492)$ & \\
Director & $161(32.7)$ \\
Managerial & $117(23.8)$ \\
All other foodservice staff & $65(13.2)$ \\
Administration & $68(13.8)$ \\
Other staff & $81(16.5)$ \\
\hline Years of experience in child nutrition: $(n=491)$ & \\
$<3$ & $73(14.9)$ \\
$3-5$ & $83(17.0)$ \\
$5-10$ & $93(18.9)$ \\
$>10$ & $242(49.3)$ \\
\hline Neighborhood area type: $(n=365)$ & $133(36.4)$ \\
Rural & $232(63.6)$ \\
\hline
\end{tabular}


Table 1. Cont.

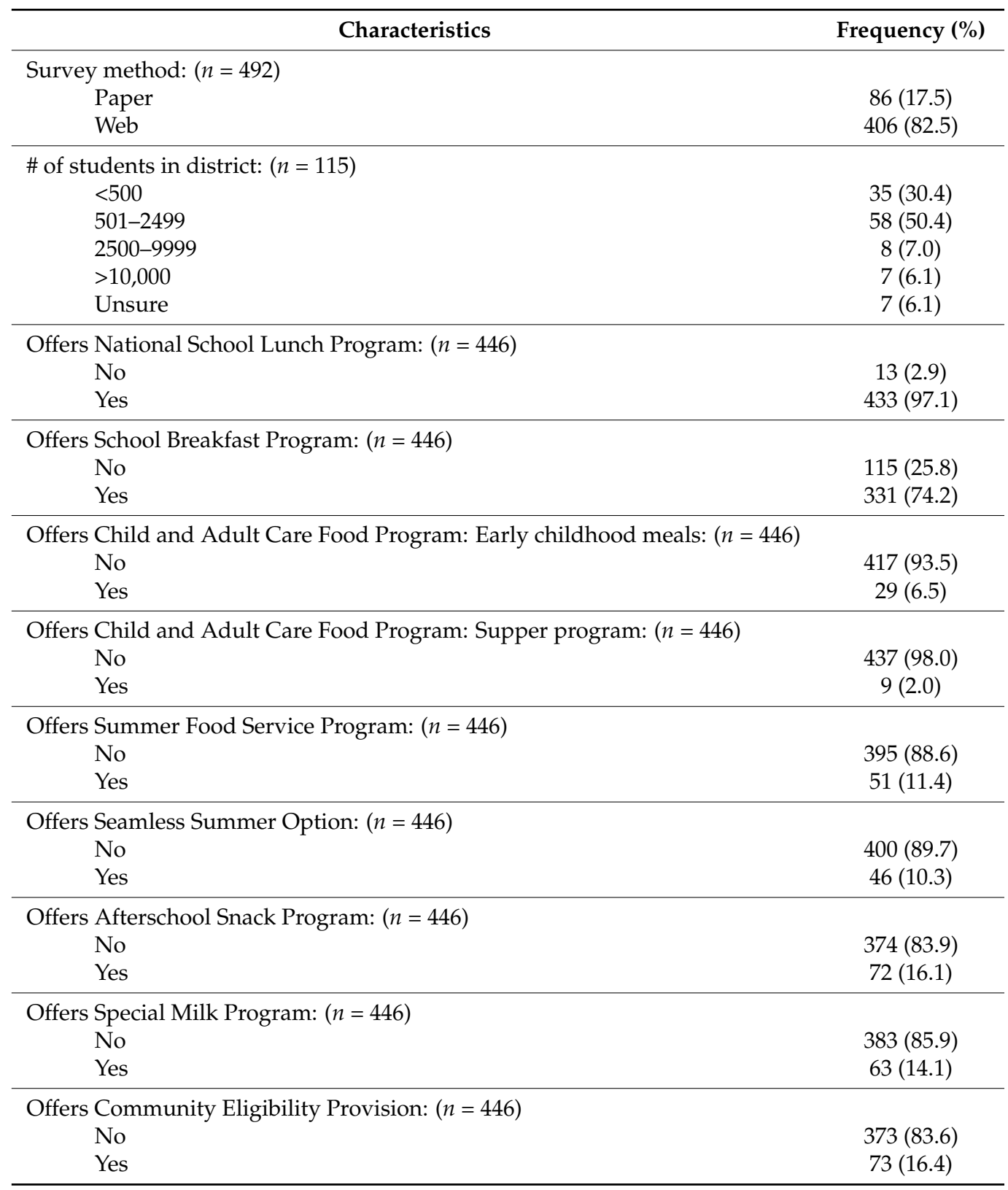

\subsection{Preferred Learning Methods}

Table 2 provides the overall results for learning method preferences. The most preferred learning methods were completing multiple activities during a training, face to face trainings, and hands-on interactive activities with 91.5\% $(n=419), 85.7 \%(n=394)$, and $85.4 \%(n=392)$ respectively rating them as "like somewhat" or "like a great deal." Conversely, role play was the least preferred learning method overall. Figure 1 shows the learning method preferences that differ by role, and Figure 2 shows the learning method preferences that differ by school locale. 
Table 2. Reported Preferred Learning Methods of Illinois Child Nutrition Program staff.

\begin{tabular}{|c|c|c|c|c|c|}
\hline \multirow{2}{*}{$\begin{array}{l}\text { Learning Preferences: } \\
\text { Like to Attend Training on/with: }\end{array}$} & \multicolumn{5}{|c|}{ Frequency (\%) } \\
\hline & $\begin{array}{l}\text { Dislike a } \\
\text { Great Deal }\end{array}$ & $\begin{array}{l}\text { Dislike } \\
\text { Somewhat }\end{array}$ & $\begin{array}{c}\text { Neither } \\
\text { Like/Dislike }\end{array}$ & $\begin{array}{c}\text { Like } \\
\text { Somewhat }\end{array}$ & $\begin{array}{c}\text { Like a } \\
\text { Great Deal }\end{array}$ \\
\hline Interactive Activity: $(n=459)$ & $9(2.0)$ & $10(2.2)$ & $48(10.5)$ & $146(31.8)$ & $246(53.6)$ \\
\hline Peer to Peer Sharing: $(n=461)$ & $13(2.8)$ & $18(3.9)$ & $82(17.8)$ & $171(37.1)$ & $177(38.4)$ \\
\hline Training by Myself: $(n=460)$ & $22(4.8)$ & $30(6.5)$ & $122(26.5)$ & $170(37.0)$ & $116(25.2)$ \\
\hline A Partner: $(n=459)$ & $7(1.5)$ & $25(5.5)$ & $100(21.8)$ & $210(45.7)$ & $117(25.5)$ \\
\hline With a Group: $(n=459)$ & $18(3.9)$ & $38(8.3)$ & $89(19.4)$ & $198(43.1)$ & $116(25.3)$ \\
\hline Multiple Activities during Training $(n=461)$ & $7(1.5)$ & $17(3.7)$ & $84(18.2)$ & $188(40.8)$ & $165(35.8)$ \\
\hline Hear from an Expert: $(n=458)$ & - & $4(0.9)$ & $35(7.6)$ & $125(27.3)$ & $294(64.2)$ \\
\hline Training Online: $(n=460)$ & $13(2.8)$ & $40(8.7)$ & $67(14.6)$ & $159(34.6)$ & $181(39.4)$ \\
\hline Face to Face Learning: $(n=460)$ & $4(0.9)$ & $5(1.1)$ & $57(12.4)$ & $160(34.8)$ & $234(50.9)$ \\
\hline Learn on Text with Many Pictures: $(n=459)$ & $29(6.3)$ & $45(9.8)$ & $144(31.4)$ & $153(33.3)$ & $88(19.2)$ \\
\hline Watching Videos: $(n=461)$ & $12(2.6)$ & $30(6.5)$ & $104(22.6)$ & $189(41.0)$ & $126(27.3)$ \\
\hline Learn by Physical Movement: $(n=459)$ & $3(0.7)$ & $15(3.3)$ & $124(27.0)$ & $170(37.0)$ & $147(32.0)$ \\
\hline Learn by Teaching others the Material: $(n=459)$ & $17(3.7)$ & $41(8.9)$ & $128(27.7)$ & $167(36.2)$ & $109(23.6)$ \\
\hline Quizzes and Knowledge Check: $(n=460)$ & $17(3.7)$ & $54(11.7)$ & $135(29.4)$ & $171(37.2)$ & $83(18.0)$ \\
\hline Worksheets: $(n=458)$ & $19(4.2)$ & $45(9.8)$ & $132(28.8)$ & $172(37.6)$ & $90(19.7)$ \\
\hline Learn with Problem-Solving or Brainstorming: $(n=460)$ & $11(2.4)$ & $20(4.4)$ & $102(22.2)$ & $184(40.0)$ & $143(31.1)$ \\
\hline Role Play or Demonstration: $(n=460)$ & $47(10.2)$ & $69(15.0)$ & $128(27.8)$ & $125(27.2)$ & $91(19.8)$ \\
\hline
\end{tabular}

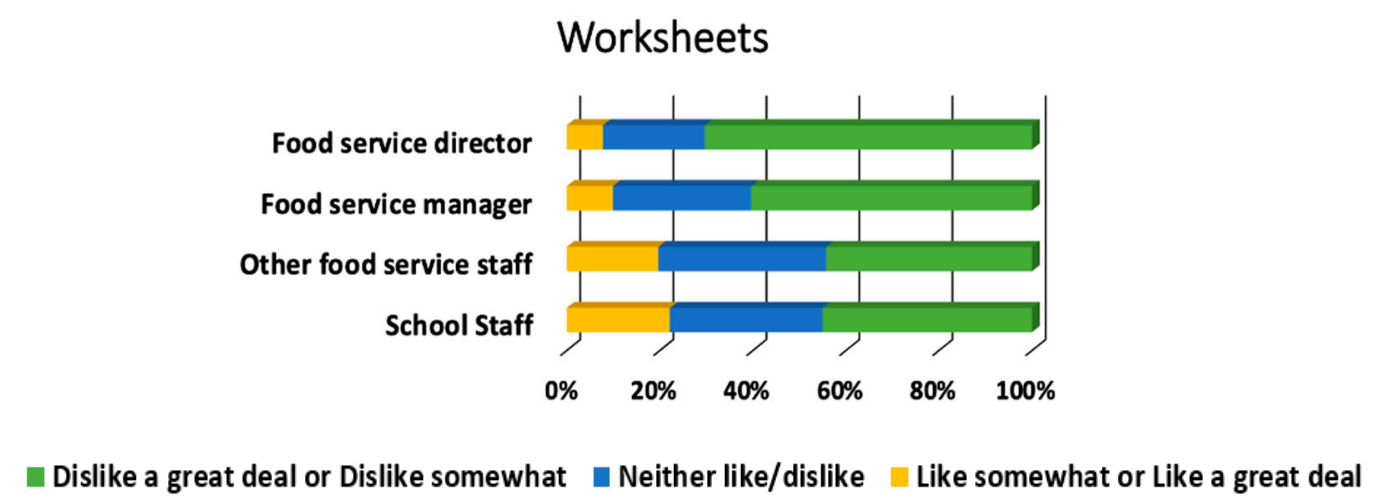

Figure 1. Preference for completing worksheets significantly differed by participant role. [ $n=458$ (food service director, $n=155$; food service manager, $n=111$; other food service staff, $n=61$; school staff, $n=131), \chi^{2}(6)=27.7, p<0.001$, Cramer's V=0.17].

\section{Teaching others the information}

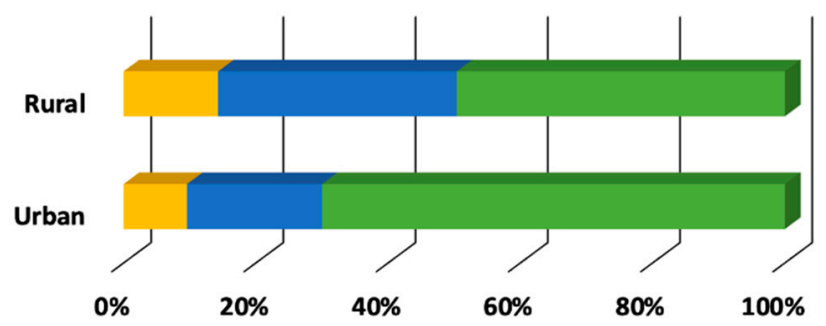

—islike a great deal or Dislike somewhat neither like/dislike — Like somewhat or Like a great deal

Figure 2. Preference for learning by teaching others significantly differed by school locale. [ $n=361$ (rural, $n=131$; urban, $n=230), \chi 2(2)=15.1, p<0.001$, Cramer's $\mathrm{V}=0.20]$. 


\subsection{Reported Training Topic Preferences}

Table 3 provides the overall results for training topics. Overall, the most desired training topics were preparing for administrative reviews, employee training, procedures, and policies, and school wellness policies with $75.5 \%(n=342), 74.83 \%(n=339)$, and $72.2 \%(n=327)$ respectively rating them as "somewhat likely" or "very likely." Conversely, there were six training topics with $25 \%$ of overall respondents rating them as "somewhat unlikely" or "unlikely to attend": meal pattern requirements, Smart Snacks, standardized recipes, meal preparation and cooking skills, meal pattern crediting documents, and product management. Nearly all of the training topics (34 out of 35) were preferred differentially by participant role $(p<0.001)$, and $85.3 \%(n=29)$ of these relationships had moderate to strong effect sizes, as shown in Figures 3-6. There was no evidence of significant differences in training topic preferences by years of job experience. Three training topics (5.6\%) were preferred differentially by locale; all three had moderate effect sizes (Figure 7).

Table 3. Reported Training Topic Preferences of Illinois Child Nutrition Program staff.

\begin{tabular}{|c|c|c|c|c|c|}
\hline \multirow[b]{2}{*}{ Training Topics } & \multicolumn{5}{|c|}{ Frequency $(\%)$} \\
\hline & $\begin{array}{c}\text { Very } \\
\text { Unlikely }\end{array}$ & $\begin{array}{l}\text { Somewhat } \\
\text { Unlikely }\end{array}$ & $\begin{array}{c}\text { Neither } \\
\text { Likely Nor } \\
\text { Unlikely }\end{array}$ & $\begin{array}{l}\text { Somewhat } \\
\text { Likely }\end{array}$ & $\begin{array}{c}\text { Very } \\
\text { Likely }\end{array}$ \\
\hline \multicolumn{6}{|c|}{ Nutrition: } \\
\hline Portion Sizes: $(n=453)$ & $71(15.7)$ & $41(9.1)$ & $78(17.2)$ & $142(31.4)$ & $121(26.7)$ \\
\hline Local Foods: $(n=453)$ & $64(14.1)$ & $40(8.8)$ & $110(24.3)$ & $139(30.7)$ & $100(22.1)$ \\
\hline Smart Snacks: $(n=453)$ & $75(16.6)$ & $39(8.6)$ & $69(15.2)$ & $157(34.7)$ & $113(24.9)$ \\
\hline USDA Foods: $(n=453)$ & $59(13.0)$ & $26(5.7)$ & $64(14.1)$ & $172(38.0)$ & $132(29.1)$ \\
\hline Special Dietary Needs: $(n=453)$ & $47(10.4)$ & $36(8.0)$ & $70(15.5)$ & $160(35.3)$ & $140(30.9)$ \\
\hline USDA Team Nutrition materials $(n=453)$ & $46(10.2)$ & $35(7.7)$ & $98(21.6)$ & $158(34.9)$ & $116(25.6)$ \\
\hline Basic Nutrition Education: $(n=453)$ & $63(13.9)$ & $33(7.3)$ & $72(15.9)$ & $142(31.4)$ & 143(31.6) \\
\hline \multicolumn{6}{|c|}{ Operations: } \\
\hline Standardized Recipes: $(n=452)$ & $86(19.0)$ & $31(6.9)$ & $71(15.7)$ & $128(28.3)$ & $136(30.1)$ \\
\hline Production Records: $(n=452)$ & $72(15.9)$ & $31(6.9)$ & $69(15.3)$ & $144(31.9)$ & $136(30.1)$ \\
\hline Meal Preparation and Cooking Skills: $(n=452)$ & $83(18.4)$ & $34(7.5)$ & $72(15.9)$ & $130(28.8)$ & $133(29.4)$ \\
\hline Meal Pattern Crediting Documents: $(n=452)$ & $79(17.5)$ & $34(7.5)$ & $76(16.8)$ & $137(30.3)$ & $126(27.9)$ \\
\hline Fruit and Vegetable Bars: $(n=452)$ & $71(15.7)$ & $40(8.9)$ & $75(16.6)$ & $138(30.5)$ & $128(28.3)$ \\
\hline Responsibilities at Point-of-Service: $(n=452)$ & $44(9.7)$ & $26(5.8)$ & $83(18.4)$ & $151(33.4)$ & $148(32.7)$ \\
\hline Procurement Strategies: $(n=452)$ & $63(13.9)$ & $45(10.0)$ & $88(19.5)$ & $114(25.2)$ & $142(31.4)$ \\
\hline Product Management: $(n=452)$ & $71(15.7)$ & $46(10.2)$ & $97(21.5)$ & $136(30.1)$ & $102(22.6)$ \\
\hline Food Safety Management: $(n=452)$ & $59(13.1)$ & $27(6.0)$ & $68(15.0)$ & $154(34.1)$ & $144(31.9)$ \\
\hline Food Safety Training: $(n=452)$ & $64(14.2)$ & $30(6.6)$ & $62(13.7)$ & $131(29.0)$ & $165(36.5)$ \\
\hline \multicolumn{6}{|c|}{ Administration: } \\
\hline Certification or Verification of Benefits: $(n=453)$ & $42(9.3)$ & $33(7.3)$ & $79(17.4)$ & $121(26.7)$ & $178(39.3)$ \\
\hline School Wellness Policies: $(n=453)$ & $27(6.0)$ & $21(4.6)$ & $78(17.2)$ & $161(35.5)$ & $166(36.6)$ \\
\hline Financial Management: $(n=453)$ & $51(11.3)$ & $42(9.3)$ & $99(21.9)$ & $112(24.7)$ & $149(32.8)$ \\
\hline $\begin{array}{l}\text { Key Performance Indicators to make Data Driven Decisions: } \\
\qquad(n=453)\end{array}$ & $42(9.3)$ & $38(8.4)$ & $86(19.0)$ & $128(28.3)$ & $159(35.1)$ \\
\hline Employee Training Procedures and Policies: $(n=453)$ & $32(7.1)$ & $17(3.8)$ & $65(14.4)$ & $166(36.6)$ & $173(38.2)$ \\
\hline Training on Employee Management: $(n=453)$ & $52(11.5)$ & $30(6.6)$ & $82(18.1)$ & $141(31.1)$ & $148(32.7)$ \\
\hline Managing Food and Packaging Waste: $(n=453)$ & $62(13.7)$ & $29(6.4)$ & $104(23.0)$ & $150(33.1)$ & $108(23.8)$ \\
\hline Preparing for Administrative Reviews: $(n=453)$ & $27(6.0)$ & $18(4.0)$ & $66(14.6)$ & $127(28.0)$ & $215(47.5)$ \\
\hline Assessing and Planning for Equipment Needs: $(n=453)$ & $52(11.5)$ & $34(7.5)$ & $109(24.1)$ & 139 (30.7) & 119 (26.3) \\
\hline
\end{tabular}


Table 3. Cont.

\begin{tabular}{|c|c|c|c|c|c|}
\hline \multirow[b]{2}{*}{ Training Topics } & \multicolumn{5}{|c|}{ Frequency (\%) } \\
\hline & $\begin{array}{c}\text { Very } \\
\text { Unlikely }\end{array}$ & $\begin{array}{l}\text { Somewhat } \\
\text { Unlikely }\end{array}$ & $\begin{array}{l}\text { Neither } \\
\text { Likely Nor } \\
\text { Unlikely }\end{array}$ & $\begin{array}{l}\text { Somewhat } \\
\text { Likely }\end{array}$ & $\begin{array}{c}\text { Very } \\
\text { Likely }\end{array}$ \\
\hline \multicolumn{6}{|c|}{ Communication and Marketing: } \\
\hline Customer Service: $(n=453)$ & $48(10.6)$ & $37(8.2)$ & $120(26.5)$ & $144(31.8)$ & $104(23.0)$ \\
\hline $\begin{array}{l}\text { Marketing Child Nutrition Programs to School and Staff: } \\
\qquad(n=453)\end{array}$ & $49(10.8)$ & $24(5.3)$ & $101(22.3)$ & $160(35.3)$ & $119(26.3)$ \\
\hline $\begin{array}{l}\text { Marketing Child Nutrition Program to Students and Families: } \\
\qquad(n=453)\end{array}$ & $40(8.8)$ & $20(4.4)$ & $92(20.3)$ & $169(37.3)$ & $132(29.1)$ \\
\hline Social Media to Promote Child Nutrition Programs: $(n=453)$ & $46(10.2)$ & $31(6.8)$ & $108(23.8)$ & $150(33.1)$ & $118(26.1)$ \\
\hline $\begin{array}{l}\text { Smarter Lunchrooms and Food Merchandising Techniques: } \\
\qquad(n=453)\end{array}$ & $51(11.3)$ & $27(6.0)$ & $91(20.1)$ & $166(36.6)$ & $118(26.1)$ \\
\hline Improving Computer Skills: $(n=453)$ & $60(13.3)$ & $32(7.1)$ & $113(24.9)$ & $130(28.7)$ & $118(26.1)$ \\
\hline Increasing Student Engagement and Involvement: $(n=453)$ & $47(10.4)$ & $18(4.0)$ & $74(16.3)$ & $155(34.2)$ & $159(35.1)$ \\
\hline
\end{tabular}

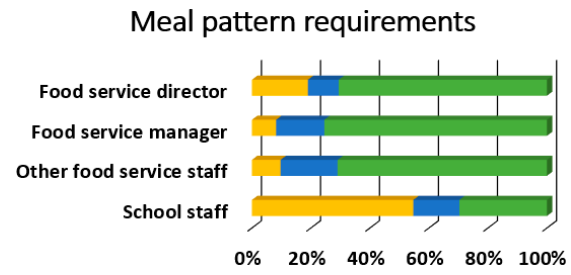

(a)

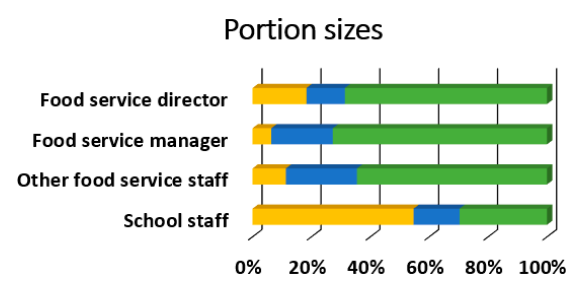

(c)

Smart Snacks

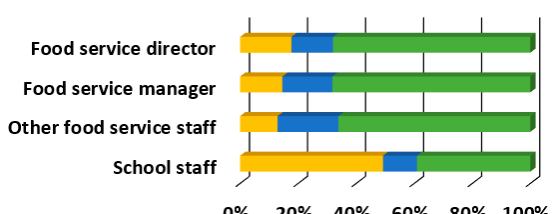

(e)

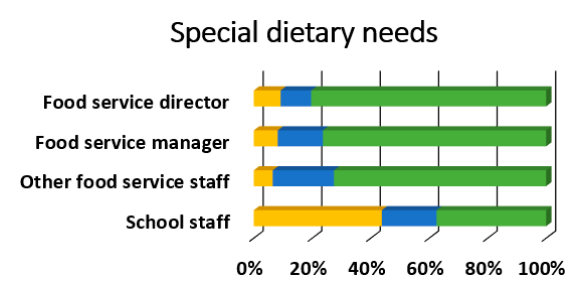

(g)

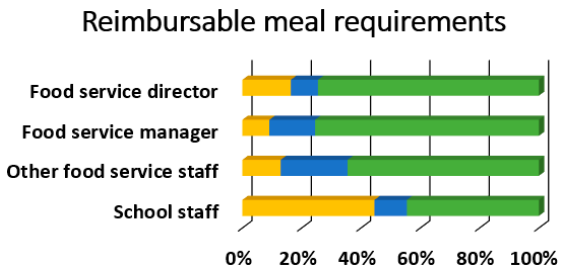

(b)

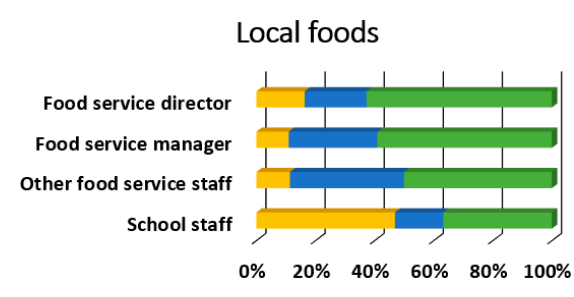

(d)

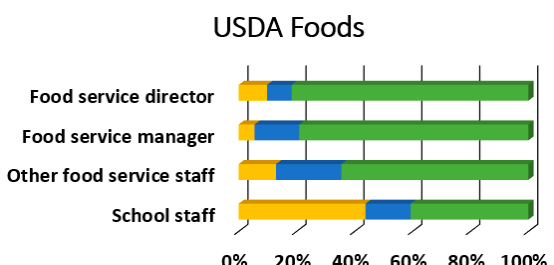

(f)

USDA Team Nutrition materials

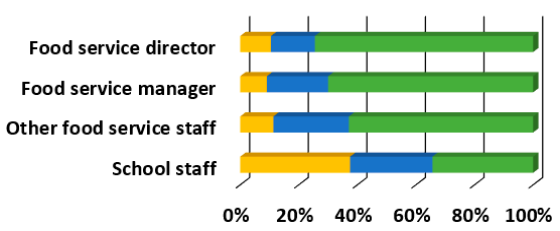

(h)

Figure 3. Cont. 


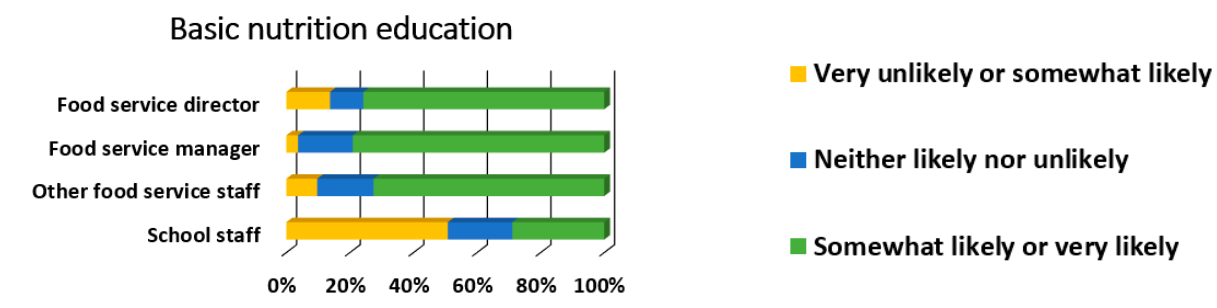

(i)

Figure 3. Nutrition training topic needs that significantly differed by participant role, $n=453$ (food service director, $n=153$; food service manager, $n=110$; other food service staff, $n=62$; school staff, $n=128$ ). Described below for each panel are the $\chi^{2}$ and $p$-values. (a) Meal pattern requirements, $\chi^{2}(6)=96.2, p<0.001$, Cramer's V $=0.33$; (b) Reimbursable meal requirements, $\chi^{2}(6)=61.6, p<0.001$, Cramer's V = 0.26; (c) Portion sizes, $\chi^{2}(6)=98.1, p<0.001$, Cramer's V=0.33; (d) Local foods, $\chi^{2}(6)=66.3, p<0.001$, Cramer's V = 0.27; (e) Smart Snacks, $\chi^{2}(6)=56.9, p<0.001$, Cramer's V = 0.25;(f) USDA foods, $\chi^{2}(6)=88.2, p<0.001$, Cramer's V = 0.31; (g) Special dietary needs, $\chi^{2}(6)=90.4, p<0.001$, Cramer's V = 0.32; (h) USDA Team Nutrition materials, $\chi^{2}(6)=65.7, p<0.001$, Cramer's V = 0.27; (i) Basic nutrition education, $\chi^{2}(6)=114.5, p<0.001$, Cramer's V $=0.36$.

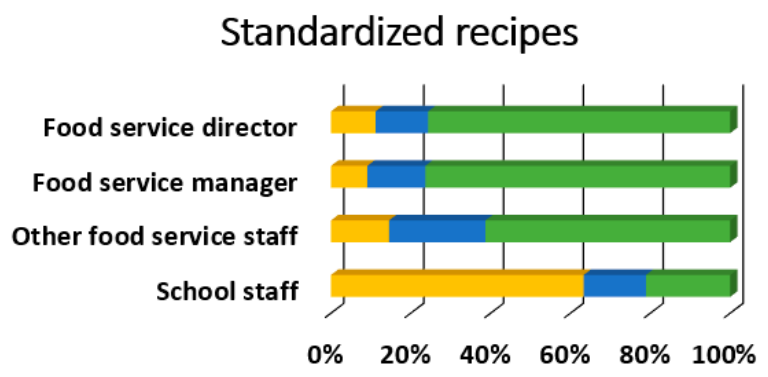

(a)

\section{Meal preparation and cooking skills}

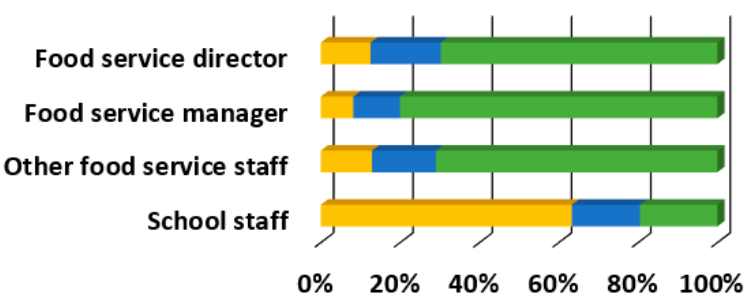

(c)

\section{Production records}

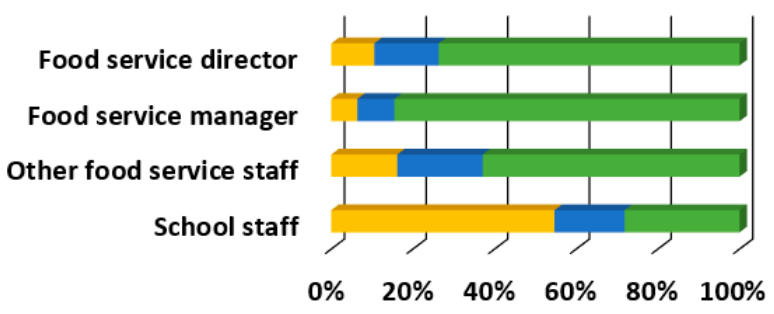

(b)

\section{Meal pattern crediting documents}

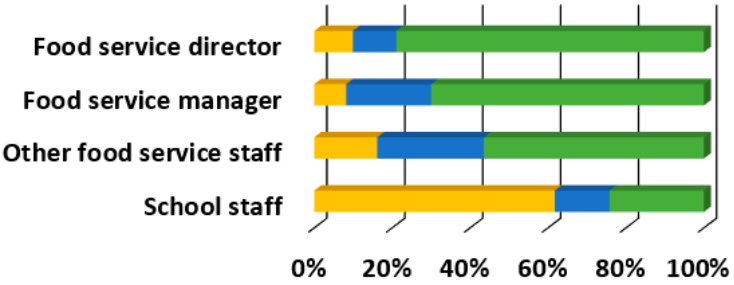

(d)

\section{Fruit and vegetable bars}

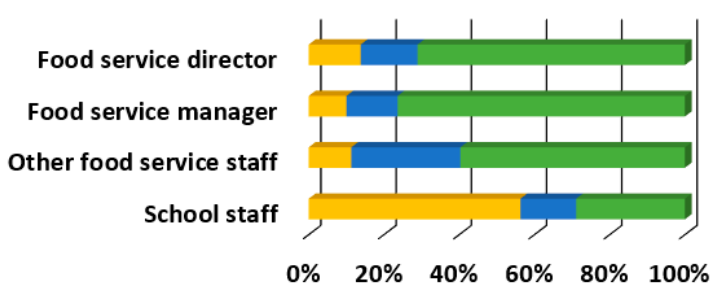

(e)

Figure 4. Cont. 


\section{Procurement strategies}

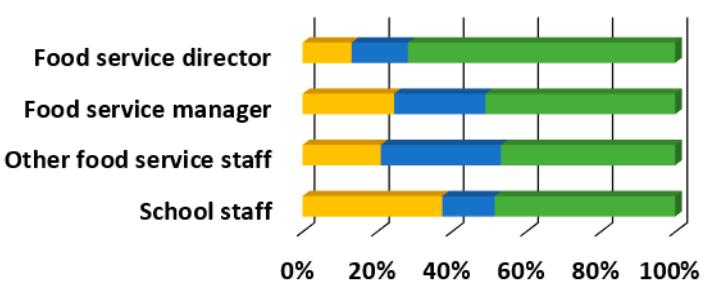

(f)

\section{Food safety management}

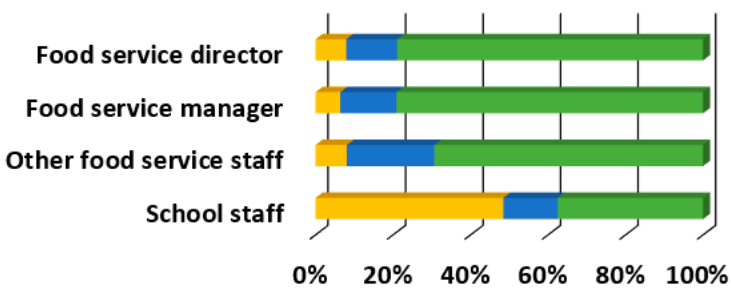

(h)

\section{Product management}

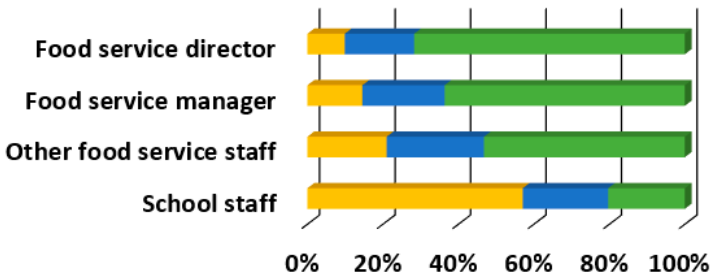

(g)

\section{Food safety training}

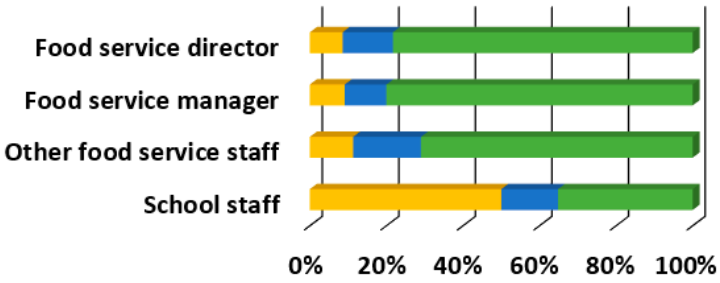

(i)

\section{Very unlikely or somewhat likely $\quad$ Neither likely nor unlikely $\quad$ Somewhat likely or very likely}

Figure 4. Operations training topic needs that significantly differed by participant role, $n=452$ (food service director, $n=152$; food service manager, $n=110$; other food service staff, $n=62$; school staff, $n=128$ ). Described below for each panel are the $\chi^{2}$ and $p$-values. (a) Standardized recipes, $\chi^{2}(6)=144.9, p<0.001$, Cramer's V = 0.40; (b) Production records, $\chi^{2}(6)=121.9$, $p<0.001$, Cramer's V = 0.37; (c) Meal preparation and cooking skills, $\chi^{2}(6)=145.7, p<0.001$, Cramer's V=0.40; (d) Meal pattern crediting documents, $\chi^{2}(6)=145.9, p<0.001$, Cramer's V =0.40; (e) Fruit and vegetable bars, $\chi^{2}(6)=109.3$, $p<0.001$, Cramer's V = 0.35; (f) Procurement strategies, $\chi^{2}(6)=36.9, p<0.001$, Cramer's V = 0.20; (g) Product management, $\chi^{2}(6)=108.8, p<0.001$, Cramer's V = 0.35; (h) Food safety management, $\chi^{2}(6)=106.7, p<0.001$, Cramer's V = 0.34; (i) Food safety training, $\chi^{2}(6)=101.0, p<0.001$, Cramer's V $=0.33$.

\section{Certification of benefits}

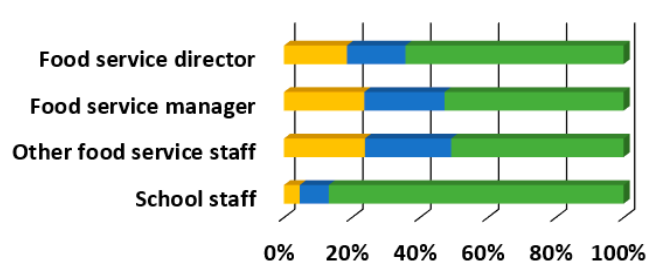

(a)

\section{Key performance indicators}

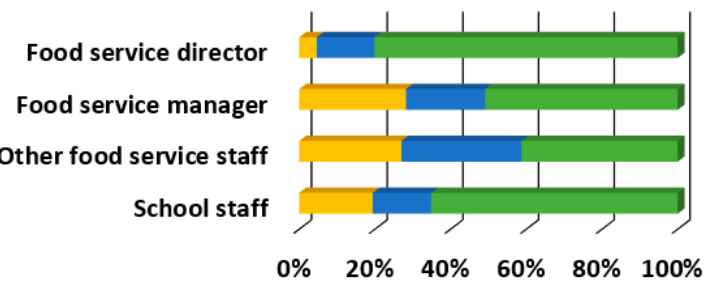

(c)

\section{Financial management}

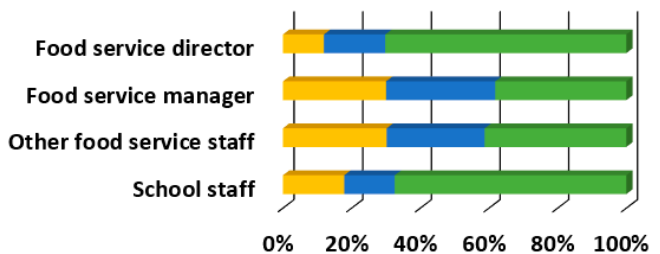

(b)

\section{Employee training}

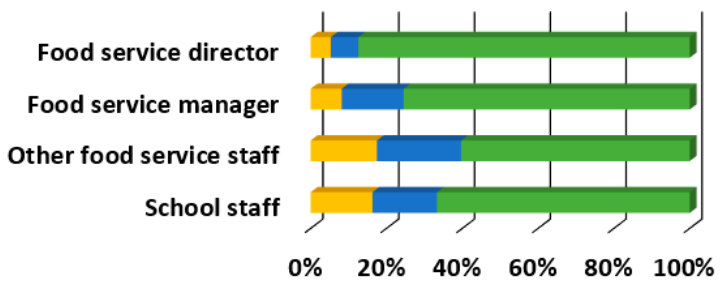

(d)

Figure 5. Cont. 


\section{Employee management}

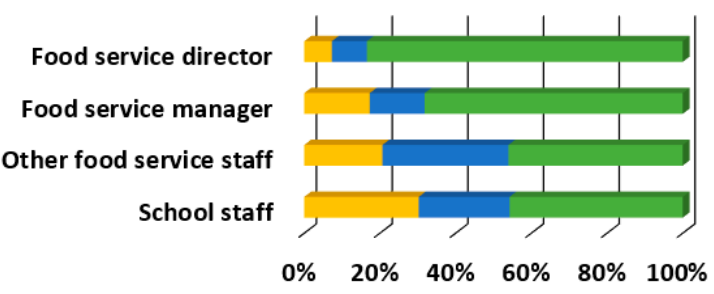

(e)

\section{Preparing for administrative reviews}

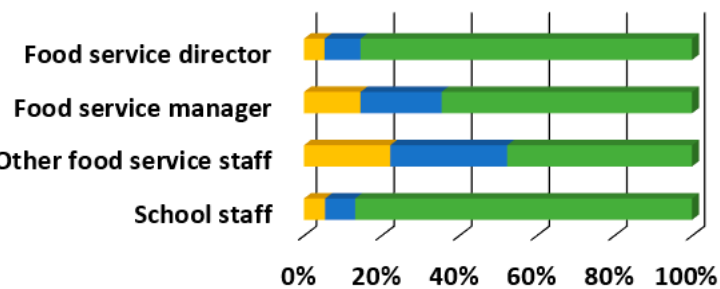

(g)

\section{Managing food and packaging waste}

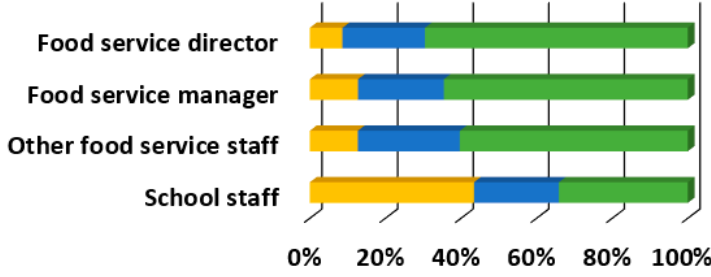

(f)

\section{Assessing equipment needs}

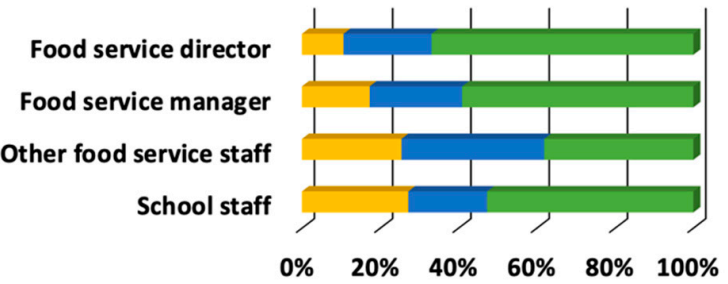

(h)

Very unlikely or somewhat likely $\quad$ Neither likely nor unlikely $\square$ Somewhat likely or very likely

Figure 5. Administration training topic needs that significantly differed by participant role, $n=453$ (food service director, $n=151$; food service manager, $n=110$; other food service staff, $n=63$; school staff, $n=129$ ). Described below for each panel are the $\chi^{2}$ and $p$-values. (a) Certification/verification of benefits (free- and reduced-price meal benefits), $\chi^{2}(6)=41.0$, $p<0.001$, Cramer's V = 0.21; (b) Financial management (budget preparation, revenue/expenditures), $\chi^{2}(6)=40.4, p<0.001$, Cramer's $\mathrm{V}=0.21$; (c) Key performance indicators to make data-driven decisions (e.g., average daily participation, meal equivalents, cost per meal equivalent, break-even point), $\chi^{2}(6)=46.6, p<0.001$, Cramer's V =0.23; (d) Employee training, procedures, and policies, $\chi^{2}(6)=26.2, p<0.001$, Cramer's V = 0.17; (e) Employee management (discipline, communication, delegating, giving praise, etc.), $\chi^{2}(6)=57.5, p<0.001$, Cramer's V = 0.25; (f) Managing food and packaging waste, $\chi^{2}(6)=67.3, p<0.001$, Cramer's V = 0.27; (g) Preparing for administrative reviews, $\chi^{2}(6)=50.8, p<0.001$, Cramer's V $=0.24 ;(\mathbf{h})$ Assessing and planning for equipment needs, $\chi^{2}(6)=23.7, p<0.001$, Cramer's V $=0.16$.

\section{Customer service}

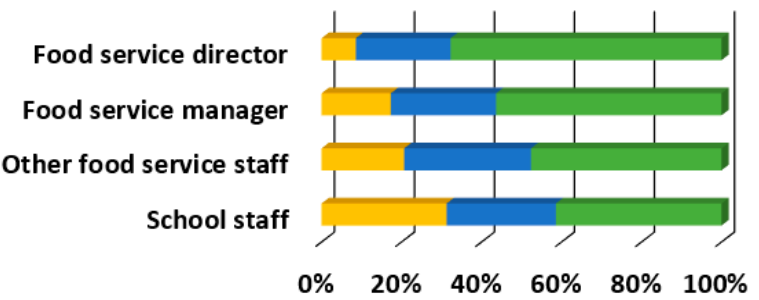

(a)

\section{Marketing CNP to school staff}

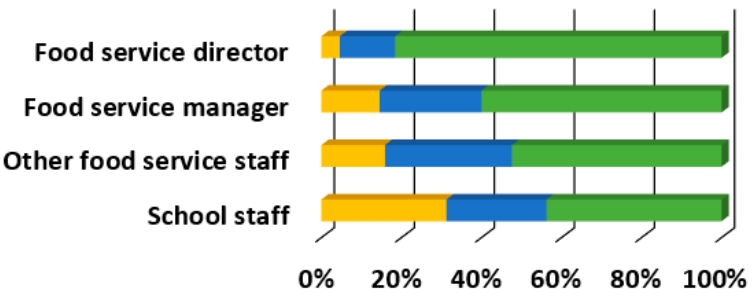

(b)

Figure 6. Cont. 
Marketing CNP to students/families

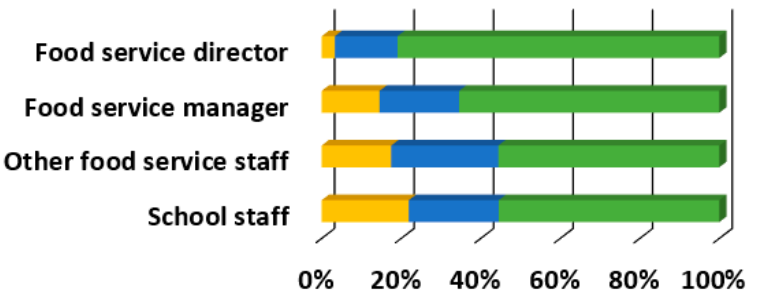

(c)

\section{Food merchandising}

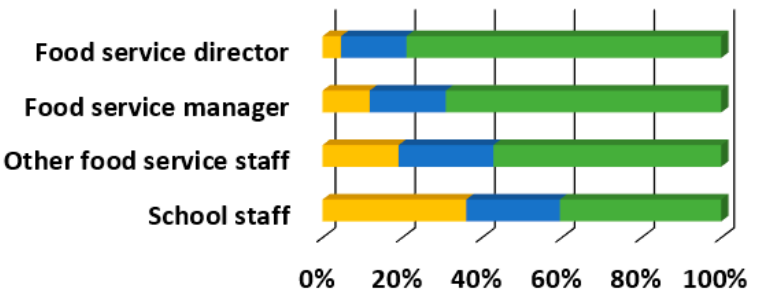

(e)
Promote CNP with social media

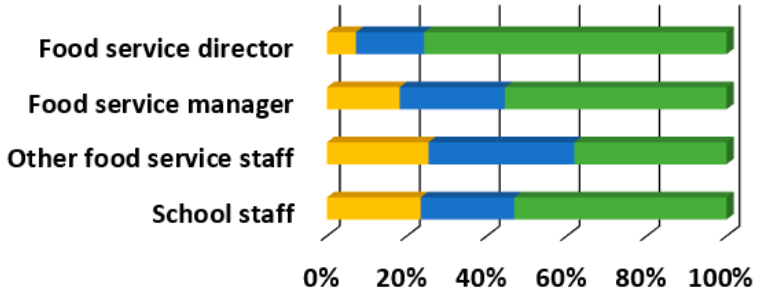

(d)

\section{Student engagement}

Food service director

Food service manager

Other food service staff

School staff

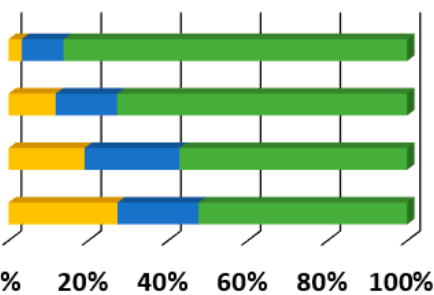

(f)

\section{Very unlikely or somewhat likely $\quad$ Neither likely nor unlikely $\quad$ Somewhat likely or very likely}

Figure 6. Communications and Marketing training topic needs that significantly differed by participant role, $n=453$ (food service director, $n=152$; food service manager, $\mathrm{n}=110$; other food service staff, $n=63$; school staff, $n=128$ ). Described below for each panel are the $\chi^{2}$ and $p$-values. CNP $=$ Child Nutrition Programs. (a) Customer service, $\chi^{2}(6)=30.0, p<0.001$, Cramer's V =0.18; (b) Marketing child nutrition programs to school staff, $\chi^{2}(6)=56.6, p<0.001$, Cramer's V = 0.25; (c) Marketing child nutrition programs to students and families, $\chi^{2}(6)=31.1, p<0.001$, Cramer's V $=0.19$; (d) Using social media to promote child nutrition programs, $\chi^{2}(6)=34.5, p<0.001$, Cramer's V = 0.20; (e) Smarter Lunchrooms and food merchandising techniques, $\chi^{2}(6)=61.7, p<0.001$, Cramer's $V=0.26$; (f) Increasing student engagement and involvement, $\chi^{2}(6)=49.2, p<0.001$, Cramer's $V=0.23$.

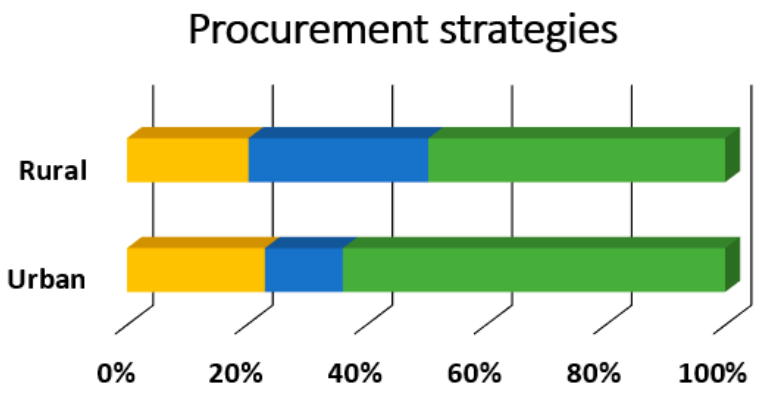

(a)

\section{Key performance indicators}

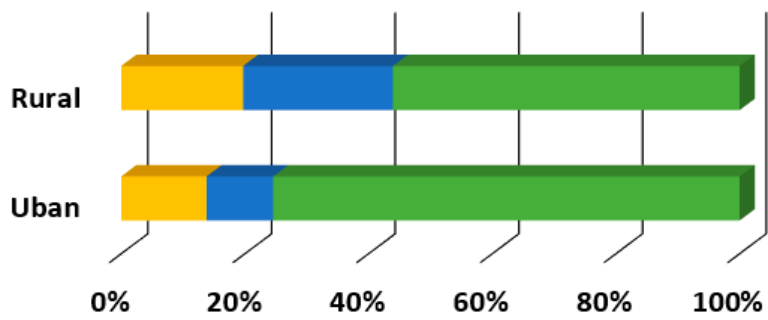

(b)

Figure 7. Cont. 


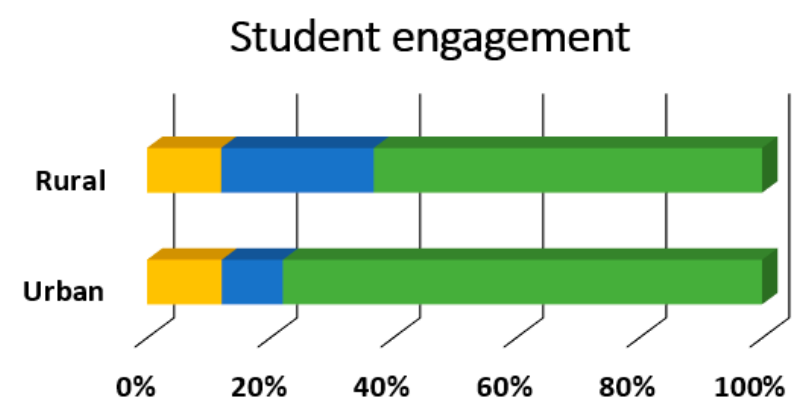

(c)

\section{Very unlikely or somewhat likely $\quad$ Neither likely nor unlikely $\quad$ Somewhat likely or very likely}

Figure 7. Nutrition, Operations, Administration, and Communications and Marketing training topic needs that significantly differed by school locale, $n=364$ (rural, $n=133$; urban, $n=231$ ). Described below for each panel are the $\chi^{2}$ and $p$-values. (a) Procurement strategies (bidding process and writing specifications, purchasing co-operatives, etc.), $\chi^{2}(2)=15.9, p<0.001$, Cramer's $\mathrm{V}=0.21$; (b) Key performance indicators to make data-driven decisions (e.g., average daily participation, meal equivalents, cost per meal equivalent, break-even point), $\chi^{2}(2)=16.2, p<0.001$, Cramer's V $=0.21$; (c) Increasing student engagement and involvement, $\chi^{2}(2)=14.6, p=0.001$, Cramer's V $=0.20$.

When broken down by participant role, the top five topics for FSWs were special dietary needs; basic nutrition education; meal pattern requirements; meal preparation and cooking skills; and food safety training. For FSMs, the top five topics were production records; meal preparation and cooking skills; food safety training; USDA foods; and basic nutrition education. The top five topics for FSDs were employee training, procedures, and policies; student engagement; preparing for administrative reviews; employee management; and USDA foods. The top five topics for other school staff were certification of benefits; preparing for administrative reviews; school wellness policies; financial management; and employee training.

\subsection{Qualitative Results from Open-Ended Respones}

After excluding non-substantive responses $(n=30)$, such as "none" or "I feel like you have covered it", there were 93 requests for training needs for the open-ended question which were submitted by FSDs $(n=36)$, FSMs $(n=15)$, FSWs $(n=6)$, school administrators $(n=17)$ and other school staff $(n=19)$. As shown in Table 4 , there were three themes present across four or more participant role groups. First, participants requested to have trainings that were targeted to specific roles or needs, such as those involved in cafeteria operations versus those whose job responsibilities focus on menu documentation, financial management, and other administrative duties. Similarly, participants requested trainings geared toward new staff as well as trainings that go beyond basic understanding to meet the needs of experienced staff. Second, participants requested innovation in programming to facilitate training access and convenience. Some responses that were included in this theme requested either online or in-person training, but there was no clear majority preference between the two mediums. One of the more common innovations requested was hearing from other staff or school nutrition experts, such as through a roundtable or multi-speaker webinar. Third, participants requested more locally available training options. 
Table 4. Qualitative analysis findings of the open-ended survey question $(n=93)^{1}$.

\begin{tabular}{|c|c|c|}
\hline Theme & $\begin{array}{l}\text { Participant Roles * } \\
\text { Included }\end{array}$ & Representative Quotes \\
\hline $\begin{array}{l}\text { Desire for role-targeted or need-specific } \\
\text { training }(n=55)\end{array}$ & $\begin{array}{l}\mathrm{FSD}=24 \\
\mathrm{FSM}=9 \\
\mathrm{FSW}=2 \\
\mathrm{SA}=8 \\
\text { Other school staff }=12\end{array}$ & $\begin{array}{l}\text { "I want to meet the annual training requirements in a way that's relevant to my job. We contract with a food service } \\
\text { management company so it doesn't make sense for me to get in the weeds on menu planning and dietary calculations, } \\
\text { but I would be very interested in the Operational and Communication training topics." (SA) } \\
\text { "New employees could use more training overall with details to review kitchen procedures, regulations and menus. } \\
\text { "(SA) } \\
\text { "Would like to see two different sessions: one for those who are actually involved in the cafeteria operations i.e., } \\
\text { managing, food preparation, serving, etc. and the other for those involved in the program application, direct } \\
\text { certification, free/reduced lunch applications, reimbursement, and verification processes. In our district, the cafeteria } \\
\text { operations are contracted with an outside agency, but I am responsible for the program application, direct certification, } \\
\text { free/reduced lunch applications, reimbursement, and verification processes. I have no need to attend a two-day } \\
\text { conference when over } 50 \% \text { of the sessions do not impact my responsibilities." (Other school staff) }\end{array}$ \\
\hline $\begin{array}{l}\text { Desire for innovation in the training mode } \\
\text { to facilitate access and convenience }(n=28)\end{array}$ & $\begin{array}{l}\mathrm{FSD}=11 \\
\mathrm{FSM}=5 \\
\mathrm{FSW}=4 \\
\mathrm{SA}=4 \\
\text { Other school staff }=4\end{array}$ & $\begin{array}{l}\text { "I think each district is so unique, but in a Director's position we are often able to leave our district with having } \\
\text { disruption in operation. Training that would allow us to attend and provide somewhat [of] a train the trainer model } \\
\text { that we can then, in turn, come back and train staff would be hugely beneficial from my perspective." (FSD) } \\
\text { "The trainings for Professional Standards hours for employees...I get that you say it is everywhere but it is not always } \\
\text { as easy as you say ... some of my staff believe it or not does not have internet access of any kind at home, and in a } \\
\text { typical work day [they] are busy from the time they clock in till the time they clock out. [Another] issue is them getting } \\
\text { upset of having more to do when [there] is only so many hours in a work day and the pay is already low scale ... They } \\
\text { feel like you just keep wanting more and more and it becomes a bit overwhelming for the pay they receive, and the } \\
\text { limited time they already have. I have literally had to give trainings to mine while we were eating lunch." (FSM) }\end{array}$ \\
\hline Desire for locally available training $(n=12)$ & $\begin{array}{l}\mathrm{FSD}=2 \\
\mathrm{FSM}=1 \\
\mathrm{FSW}=0 \\
\mathrm{SA}=5 \\
\text { Other school staff }=4\end{array}$ & $\begin{array}{l}\text { "Because the guidelines change, students' acceptance of new foods change, nutritional needs change, I feel it is } \\
\text { important for the state to offer training in various categories of nutrition. And offer it in areas throughout the state... } \\
\text { Many rural schools (probably the ones that have the least amount of help with their nutrition issues) cannot always } \\
\text { travel } 200 \text { miles for a } 4 \mathrm{~h} \text { training. Perhaps if there were trainers located throughout the state that could hold mini } \\
\text { seminars in different areas (quadrants) of the state, a larger food service population could be served." (FSD) } \\
\text { "I think training should be done locally, not just in Springfield." (FSD) } \\
\text { "We love all the training that there can be but, it would be nice if some of it was held closer to where we live like } \\
\text { Rockford or Freeport or Sterling. We cannot all travel to Springfield all the time. Or into the Chicago area. It would be } \\
\text { nice to be noticed as a small district here in Northern Illinois /Northwest Illinois. (FSM) }\end{array}$ \\
\hline
\end{tabular}

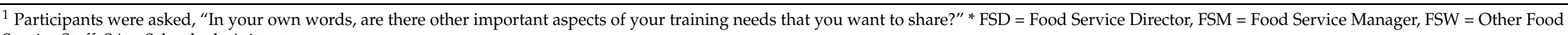
Service Staff, SA = School administrators 


\subsection{Other Training Preferences}

Participants reported that June was their most preferred month to attend trainings, while December was the least desired month. The most desired days of the week for trainings were Tuesdays, Thursdays, and weekdays when there is no school (planning days, breaks, etc.). The most preferred training duration was $1-2 \mathrm{~h}$, while greater than $4 \mathrm{~h}$ was least preferred.

\section{Discussion}

This study examined the training needs of SNPs related to USDA Professional Standards requirements and federal rules for school meals. The results suggest an important relationship between SNPs' role and preferences for training topics and learning methods, which was underscored by requests for role-targeted training in the qualitative analysis. School locale was also identified as a potential factor to be considered during training development. Although much of the variation in topic preferences appeared to be driven by responses from other school staff, there were clear differences among SNPs involved in the day-to-day operation of school meal programs and these aligned with typical job duties. For example, FSWs reported they were more likely to attend trainings which clustered in the Nutrition and Operations domains and included meal pattern requirements, special dietary needs, basic nutrition education, meal preparation and cooking skills, and food safety training. These matched well with topics that supervisors have reported as important for their staff [22] and topics that FSWs nationwide have most commonly selected as essential [23].

One interesting finding was that FSWs did not rate customer service highly, despite the fact that staff like servers and cashiers interact with students on a daily basis. If FSWs are less likely to seek out training specific to the topic, customer service exercises could be incorporated into other professional development opportunities they are likely to attend. Notably, a significant number of FSDs said they were not likely to attend trainings on the topics of meal pattern requirements, reimbursable meals, and portion sizes. Considering past research has documented significant concern among FSDs about implementing school meal standards $[2-5,8-10,22,23,25-27]$ after they went into effect, it appears that some have become more comfortable with the new nutrition standards over time. This may also be related to relaxation of requirements for sodium, milk, and whole grains during that were finalized by the USDA in 2018, which has reduced pressure on FSDs to balance nutrient requirements with product availability, cost, and student acceptance [37].

There was no evidence of a significant difference in preference for online or face-to-face learning by school locale. This contrasts with previous studies reporting that poor internet access can make it difficult for rural SNPs to access online training [5,27]. At the same time, several respondents noted in the qualitative analysis that lack of internet access was an issue. Thus, it is possible that internet connectivity in rural regions has generally improved but certain areas still lag behind. Efforts should be made to offer face-to-face training regardless of location, especially because this was one of the top ranked learning methods for the general sample. Further, they should include hands-on activities, which were highly preferred regardless of role. Topics relevant to FSWs do not necessarily require an outside expert, and they may prefer to learn in the kitchen environment where they will put such information into practice.

In terms of planning and logistics, our results indicate that trainings of $1-2 \mathrm{~h}$ were most preferred. We did not ask SNPs about training frequency, but others have reported a preference for training up to 11 times per year [20]. Thus, it may be better received and more effective for SNPs to engage in shorter trainings, perhaps on a monthly basis, or slightly longer trainings every few months. Others have suggested that professional development be offered during the work week and ideally during the regular workday [29]. However, this may not be possible due to constraints of daily meal service and many programs lack funds to pay staff overtime for training outside of normal work hours. Thus, trainings should be delivered in flexible, innovative ways, which was a major theme 
in the qualitative analysis. Qualitative responses also highlighted the desire for trainings to be regionally accessible, so they should also be offered at convenient locations to minimize time and funds needed for travel.

One limitation of the current study is that age was not considered as a potential factor impacting learning preferences. SNPs from older generations have been shown to prefer face-to-face training rather than online [20], so this should be included in future studies. The study did not investigate how demographic factors could interact; for example, FSWs in urban districts may have different needs than those in rural districts, especially if they have varying levels of experience. Preferences for training topics and learning methods could be further narrowed with additional statistical analyses or by focusing recruitment on more specific audiences.

It was challenging to obtain significant representation from FSWs, who made up about $13 \%$ of respondents. This was greater than the $8 \%$ represented in Jones et al.'s study [22], but similar to the $14 \%$ response rate for FSWs in a nationwide survey conducted by the Pew Charitable Trusts and the Robert Wood Johnson Foundation [23]. Our survey was initially sent out through electronic communications; when the research team noticed that primarily supervisors and administrators were completing it, Extension educators were asked to distribute paper copies during on-site trainings. It is possible that educators did not ask SNPs to complete the survey at trainings, or they may have neglected to mail collected surveys to the research team. Also, the majority of these trainings were conducted at smaller schools with fewer SNPs. Additional responses from this subset could be elicited by having surveys available at larger trainings, such as the state board of education's annual back-to-school nutrition conference. Surveys could also be completed directly through the Qualtrics website on laptops or tablet devices to streamline data collection. Different incentives may also be needed to motivate FSWs to complete the survey, since the marketing banners we offered as drawing prizes may have appealed more to FSMs and FSDs.

In the future, specific efforts should also be made to obtain a more geographically diverse sample. Due to limitations in recruitment, the results may not apply to all SNPs within the state. Further, our study was conducted in the Midwest and cannot necessarily be generalized to SNPs in different regions of the United States.

It should be noted that preferences were rated based on likelihood of attending training on a given topic and may better reflect training wants rather than true needs. This could be addressed in future studies by asking SNPs more specifically to list topics for which they need training or have them rate topics by importance. Further, reported training needs could be compared to administrative review reports to investigate whether perceptions of training needs match with commonly cited issues across school nutrition programs. For the current study, we did not evaluate the effectiveness of various learning methods for school nutrition training, but future research may investigate whether preferred learning methods are also more effective in terms of knowledge retention and implementation.

\section{Conclusions}

This study adds to the body of literature regarding training needs of SNPs and importantly, contributes more specific information about learning preferences depending on various factors. Based on our results, professional development for SNPs should mostly be conducted in-person, be easily accessible, include hands-on activities. Training should also be tailored by job role and address unique situational barriers. Online learning may serve as a useful adjunct for those who prefer to work independently, are comfortable with technology, and have reliable internet access.

Author Contributions: Conceptualization, L.F., W.A., M.P.P. and J.M.; literature review, L.F. and T.A.; methodology, L.F., W.A., and M.P.P.; formal analysis, W.A., and M.P.P.; resources, M.P.P. and J.M.; data curation, L.F., W.A., and M.P.P.; writing—original draft preparation, L.F., and M.P.P.; writing—review and editing, L.F., W.A., M.P.P., T.A. and J.M.; visualization, M.P.P. All authors have read and agreed to the published version of the manuscript. 
Funding: This research received no external funding.

Acknowledgments: We appreciate the diligent efforts of Feifei Chen, Elizabeth Gutierrez, Katherine Hinojosa, and Eva Liu in making the tables and figures. We would also like to acknowledge Prachi Patel and Carmen Chu for their involvement in the data cleaning.

Conflicts of Interest: The authors declare no conflict of interest.

\section{References}

1. Nutrition Standards in the National School Lunch and School Breakfast Programs. 77 Fed. Reg. 4088 (26 January 2012). Available online: https:/ / www.federalregister.gov/documents/2012/01/26/2012-1010/nutrition-standards-in-the-nationalschool-lunch-and-school-breakfast-programs (accessed on 30 October 2020).

2. Tabak, R.G.; Moreland-Russell, S. Food service perspectives on national school lunch program implementation. Health Behav. Policy Rev. 2015, 2, 362-371. [CrossRef] [PubMed]

3. Serving Healthy School Meals: Despite Challenges, Schools Meet USDA Meal Requirements; Pew Research Center: Washington, DC, USA, 2013; Available online: https://www.pewtrusts.org/-/media/legacy/uploadedfiles/phg/content_level_pages/reports/ servinghealthyschoolmealspdf.pdf (accessed on 15 October 2020).

4. School Meal Programs Innovate to Improve Student Nutrition: Survey Explores Progress; Challenges Three Years into Transition to Healthier Food Standards; Pew Research Center: Washington, DC, USA, 2016; Available online: https: / /www.pewtrusts.org/- / media/assets/2016/12/school_meal_programs_innovate_to_improve_student_nutrition.pdf (accessed on 15 October 2020).

5. Cornish, D.; Askelson, N.M.; Golembiewski, E. Reforms looked really good on paper: Rural food service responses to the healthy, hunger-free kids act of 2010. J. Sch. Health 2016, 86, 113-120. [CrossRef] [PubMed]

6. Roth-Yousey, L.; Barno, T.; Caskey, M.; Asche, K.; Reicks, M. Whole-grain continuing education for school foodservice personnel: Keeping kids from falling short. J. Nutr. Educ. Behav. 2009, 41, 429-435. [CrossRef] [PubMed]

7. Chu, Y.L.; Orsted, M.; Marquart, L.; Reicks, M. School foodservice personnel's struggle with using labels to identify whole-grain foods. J. Nutr. Educ. Behav. 2012, 44, 76-84. [CrossRef] [PubMed]

8. Alcaraz, B.; Cullen, K.W. Cafeteria staff perceptions of the new USDA school meal standards. J. Child. Nutr. Manag. 2014, 38, 1-12.

9. Thiagarajan, K.; Getty, V.M.; Johnson, H.L.; Case, M.; Herr, S.J. Methods and challenges related to implementing the new National School Lunch Program regulations in Indiana. J. Child. Nutr. Manag. 2015, 39, 1-11.

10. Yon, B.A.; Amin, S.A.; Taylor, J.C.; Johnson, R.K. School nutrition directors' perspectives on preparing for and implementing USDA's new school meal regulations. J. Child. Nutr. Manag. 2016, 40, 1-11.

11. Professional Standards for State and Local School Nutrition Programs Personnel as Required by the Healthy, Hunger-Free Kids Act of 2010. 80 Fed. Reg. 11077 (2 March 2015). Available online: https://www.federalregister.gov/documents/2015/03/02/2 015-04234/professional-standards-for-state-and-local-school-nutrition-programs-personnel-as-required-by-the (accessed on 30 October 2020).

12. Oakley, C.B.; Knight, K.; Hobbs, M.; Dodd, L.M.; Cole, J. Delivery and evaluation of training for school nutrition administrators and managers on meeting special food and nutrition needs in the school setting. J. Child. Nutr. Manag. 2011, 35, 1-16.

13. Lee, Y.M.; Kwon, J.; Sauer, K. A focus group study of child nutrition professionals' attitudes about food allergies and current training practices. J. Child. Nutr. Manag. 2014, 38, 1-14.

14. Lee, Y.M.; Kwon, J.; Sauer, K.; Ming, L.Y.; Junehee, K.; Kevin, S. Child nutrition professionals' attitudes, perceived challenges, and training related to food allergies. Health Behav. Policy Rev. 2016, 3, 165-175. [CrossRef]

15. Renn, L.; Rainville, A.J. School nutrition training needs regarding food sensitivities and food trends. J. Child. Nutr. Manag. 2019, $43,1-5$.

16. Kourosh, A.; Nsobundu, C.K.; Khosla, R.; Guffey, D.; Minard, C.G.; Levinson, A.J.; Davis, C.M. The effects of school staff food allergy education in a large urban school district. Health Behav. Policy Rev. 2020, 7, 238-247. [CrossRef]

17. Cohen, J.F.W.; Rimm, E.B.; Austin, S.B.; Hyatt, R.; Kraak, V.I.; Economos, C.D. A food service intervention improves whole grain access at lunch in rural elementary schools. J. Sch. Health 2014, 84, 212-219. [CrossRef] [PubMed]

18. Bean, M.K.; Theriault, E.; Grigsby, T.; Stewart, M.D.; LaRose, J.G. A Cafeteria personnel intervention to improve the school food environment. Am. J. Health Behav. 2019, 43, 158-167. [CrossRef] [PubMed]

19. Nothum, A.; Eggett, D.; Stokes, N. Confidence of hourly school nutrition employees with local and farm-fresh produce. J. Child. Nutr. Manag. 2019, 43, 1-16.

20. Arendt, S.W.; Roberts, K.R.; Strohbehn, C.; Arroyo, P.P.; Ellis, J.; Meyer, J. Motivating foodservice employees to follow safe food handling practices: Perceptions from a multigenerational workforce. J. Hum. Resour. Hosp. Tour. 2014, 13, 323-349. [CrossRef]

21. Stephens, L.; Shanks, C.B.; Roth, A.; Bark, K. Montana Cook Fresh workshop pilot: A K-12 school nutrition professional training to incorporate whole foods in school meals. J. Child. Nutr. Manag. 2015, 40, 1-14.

22. Jones, A.M.; Punia, M.; Young, S.; Huegli, C.C.; Zidenberg-Cherr, S. Training needs of personnel employed in programs participating in the National School Lunch Program in California. J. Child. Nutr. Manag. 2013, 37, 1-16.

23. Serving Healthy School Meals: Staff Development and Training Needs; Pew Research Center: Washington, DC, USA, 2015; Available online: https://www.pewtrusts.org/ \{\}/media/assets/2015/08/serving_healthy_school_meals_report.pdf (accessed on 15 October 2020). 
24. Federal Education Policy in Rural America. Available online: http://www.rociidaho.org/wp-content/uploads/2015/01/ROCI_ 2014FedEdPolicy_FINAL_0115.pdf (accessed on 19 October 2020).

25. Peer and Community Networks Drive Success in Rural School Meal Programs: Challenges and Strategies for Meetings Students' Nutritional Needs in Remote Areas; Pew Research Center: Washington, DC, USA, 2017; Available online: https://www.pewtrusts.org/- / media/assets /2017/10/kshf_peer_and_community_networks_drive_success_in_rural_school_meal_programs.pdf (accessed on 19 October 2020).

26. Asada, Y.; Ma, S.M.; Chriqui, J.F. Addressing equity in rural schools: Opportunities and challenges for school meal standards implementation. J. Sch. Health 2020, 90, 779-786. [CrossRef]

27. Hoffman, V.; Srinivasan, M.; Levin, M.; Scarmo, S. Operating school meal programs in rural districts: Challenges and solutions. J. Child. Nutr. Manag. 2018, 42,1-11.

28. Strohbehn, C.; Jun, J.; Arendt, S. School foodservice employees' perceptions of practice: Differences by generational age and hours worked. J. Nutr. Child. Manag. 2014, 38, 1-21.

29. Stephens, L.; Shanks, C.B. K-12 school food service staff training interventions: A review of the literature. J. Sch. Health 2015, 85, 825-832. [CrossRef] [PubMed]

30. Geverdt, D. Education Demographic and Geographic Estimates Program. (EDGE): Locale Boundaries User's Manual (NCES 2016-012); U.S. Department of Education: Washington, DC, USA, 2015. Available online: https://nces.ed.gov/programs/edge/docs/ NCES_LOCALE_USERSMANUAL_2016012.pdf (accessed on 30 October 2020).

31. Collins, J. Education techniques for lifelong learning: Principles of adult learning. Radiographics 2004, 24, 1483-1489. [CrossRef] [PubMed]

32. U.S. Department of Agriculture. Guide to Professional Standards for School Nutrition Programs. Available online: https://fns-prod. azureedge.net/sites/default/files/resource-files/ps_guide-highres.pdf (accessed on 30 October 2020).

33. Chi-Square and Cramer's V: What do you expect? In Statistics for Political Analysis: Understanding the Numbers; SAGE Publications: Thousand Oaks, CA, USA, 2017; pp. 245-272.

34. Braun, V.; Clarke, V. Using thematic analysis in psychology. Qual Res. Psychol 2006, 3, 77-101. [CrossRef]

35. Braun, V.; Clarke, V. Thematic analysis. In APA handbooks in Psychology ${ }^{\circledR}$. APA Handbook of Research Methods in Psychology. Research Designs: Quantitative, Qualitative, Neuropsychological, and Biological; Cooper, H., Camic, P.M., Long, D.L., Panter, A.T., Rindskopf, D., Sher, K.J., Eds.; American Psychological Association: Washington, DC, USA, 2012; Volume 2, pp. 57-71.

36. Thomas, D.R. A General inductive approach for analyzing qualitative evaluation data. Am. J. Eval. 2006, 27, 237-246. [CrossRef]

37. Preserve USDA's 2018 Final Rule Child Nutrition Programs: Flexibilities for Milk, Whole Grains and Sodium Requirements Fact Sheet. School Nutrition Association. 2020. Available online: https://schoolnutrition.org/uploadedFiles/Legislation_and_Policy/ SNA_Policy_Resources/2020-Flexibility-Fact.pdf (accessed on 30 October 2020). 